\title{
A FPGA implementation of GMM MUD algorithm for space formation aircraft communication system
}

\author{
Bo $\mathrm{Ma}^{1,2}$, Mingdong $\mathrm{Xu}^{1, *}$ and Zhilu $\mathrm{Wu}^{1}$ \\ ${ }^{1}$ School of Electronics and Information Engineering, Harbin Institute of Technology, Harbin, China \\ ${ }^{2}$ China Spacesat Co. Ltd., Beijing, China
}

Keywords: DS-UWB, GMM, MUD, FPGA Implementation.

\begin{abstract}
Space formation aircraft communication system needs to meet strong anti-interference ability, low power consumption and high transmission rate performance. Ultra-wideband (UWB) has high data transfer rate, low device complexity, high interference resistance and confidentiality. Therefore, UWB technology is used in space formation aircraft communication system, which is direct sequence ultra-wideband (DS-UWB) space formation aircraft communication system. This paper, we put forward a multi-user detection (MUD) algorithm based on Gaussian Mixture Model (GMM) in DS-UWB communication system and its fieldprogrammable gate array (FPGA) implementation. We adopt a way which based on MATLAB and FPGA platform, and results show that MUD algorithm based on GMM has better bit error rate (BER) performance compared to classical multi-user detection algorithm.
\end{abstract}

\section{Introduction}

The key of space formation aircraft to achieve the interconnection between the various aircraft in the formation is establishing inter-satellite links (ISL) [1]. As a multi-user system, the space formation aircraft communication system focus on the multiple-access communication technology [2]. The direct-sequence ultra-wideband system (DS-UWB) $[3,4,5]$ has aroused widely concern as a UWB multiple-access communication system that is currently being studied. However, the multiple-access interference (MAI) problem which limited the system capacity and performance [6]. Hence, multi-user detection (MUD) [7] is proposed to suppress the impact of MAI and improve the performance in DS-CDMA system. There are many MUD algorithms with excellent performance, such as matched filter detector (MF), optimum multi-user detector (OMD) [8], decorrelation multi-user detector (DEC) [9], minimum mean square error multi-user detector (MMSE) [10] and artificial intelligence algorithms.

\footnotetext{
* Corresponding author: 18b905032@stu.hit.edu.cn
} 
In this paper, we present a field-programmable gate array (FPGA) implementation of the MUD detector based on Gaussian Mixture Model (GMM). In terms of the bit error rate (BER), it's significantly better than MF, slightly better than MMSE and DEC, and approximates OMD. In addition, system has lower computational complexity and larger capacity.

The remainder of the paper is organized as follows: Section II describes the signal model, the channel model and the receiver model of DS-UWB communication system. Section III introduces the FPGA Implementation of GMM MUD algorithm. Section IV presents the implementation results. Finally, Section V concludes our paper.

\section{DS-UWB system model}

\subsection{DS-UWB signal model}

DS-UWB system transmitter model block diagram is shown in Fig.1. Initially, encode the source data into binary baseband information bits. The pseudo-random code is then used to encode the baseband bits to distinguish different users in the system. Each encoded baseband information bit will be represented as a series of repetitive pseudo-random code sequences. Finally, the pulse former generates ultra-wideband pulses.

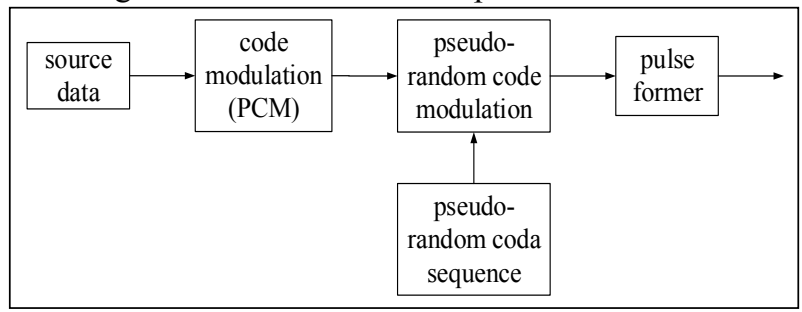

Fig. 1. DS-UWB system transmitter model block diagram.

The kth user's transmit signal of the DS-UWB system can be written as [11]:

$$
s^{(\mathrm{k})}(t)=\sum_{j=-\infty}^{\infty} \sum_{i=0}^{N_{\mathrm{s}}-1} d_{\mathrm{j}}^{(\mathrm{k})} c_{\mathrm{i}}^{(\mathrm{k})} p\left(t-i T_{\mathrm{c}}-j T_{f}\right)
$$

We assume there is a DS-UWB system with $K$ active users, employing binary phase-shift key (BPSK) modulation. Where $d_{j}{ }^{(k)} \in\{-1,+1\}$ denotes the baseband information bit of the $k$ th user, $c_{i}^{(k)} \in\{-1,+1\}$ denotes the symbol corresponding to the $i$ th chip in the coded pseudo-random sequence of user $k,\left\{c_{i}^{(\mathrm{k})}{ }_{k=0,1, \ldots, N s-1}\right\}$ denotes the address code of user $k, T_{c}$ denotes the pulse repetition period, $T_{f}$ denotes the chip period. We consider that each BPSK symbol can be divided into $N_{s}$ chips.

\subsection{DS-UWB channel model}

Considering the environment of space formation aircraft in outer space, the effects of multipath can be omitted. Furthermore, the main noise interference is approximately additive white Gaussian noise. Therefore, the channel model is an Additive White Gaussian Noise (AWGN) channel.

The electromagnetic wave propagation loss model can be defined as: 


$$
S_{R}(f)=S_{T}(f) G_{t} G_{r}\left(\frac{c}{4 \pi d f}\right)^{2}
$$

where $S_{R}(f)$ denotes received signal power spectral density, $S_{T}(f)$ denotes transmit signal power spectral density, Gt denotes the gain of the transmitter antenna and Gr denotes the gain of the receiver antenna, $c$ denotes the speed of light, $d$ denotes the distance between the receiver antenna and the transmitter antenna.

It can be known that the signal-to-noise ratio (SNR) of the receiver can be expressed as:

$$
S_{N} R_{R}(f)=\frac{S_{R}(f)}{N_{0}}=\frac{1}{N_{0}} S_{T}(f) G_{t} G_{r}\left(\frac{c}{4 \pi d f}\right)^{2}
$$

where $N_{0}=k_{B} T$ denotes power spectral density of noise in free space AWGN channels in outer space, $T$ denotes absolute temperature, $k_{B}$ denotes boltzmann constant $(1.38 \times 10-23 \mathrm{~J} / \mathrm{K})$.

It is assumed that the power spectral density of the transmitted signal is constant regardless of $S_{T}$ and frequency, we can obtain:

$$
\left\{\begin{array}{l}
S_{N R}(f)=\frac{a^{2}}{f^{2}} \\
a^{2}=\frac{1}{N_{0}} S_{T} G_{t} G_{r}\left(\frac{c}{4 \pi d}\right)^{2}
\end{array}\right.
$$

where $f_{h}$ denotes UWB signal upper cutoff frequency, $f_{l}$ denotes UWB signal lower cutoff frequency.

\subsection{DS-UWB receiver model}

DS-UWB receiver model block diagram is shown in Fig.2.

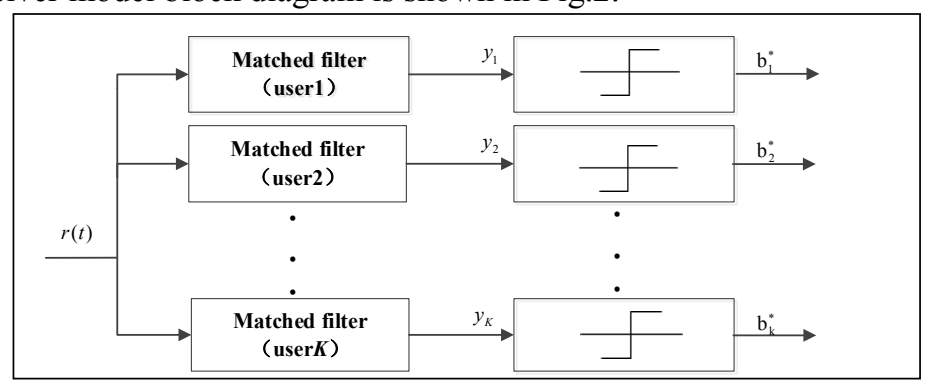

Fig. 2. DS-UWB receiver model block diagram.

We assume that the system has $\mathrm{K}$ users and the signal received by the receiver can be expressed as:

$$
r(t)=\sum_{k=1}^{K} \alpha_{k} \sqrt{P_{k}} d_{i}^{(k)} \sum_{j=0}^{N_{s}-1} c_{j}^{(k)} p\left(t-i T_{f}-j T_{c}-\tau_{k}\right)+n(t), t \in\left[i T_{f},(i+1) T_{f}\right]
$$

where $\alpha_{k}$ denotes the signal gain of the $k$ th user, $P_{k}$ denotes the signal power of user $k, \tau_{k}$ denotes the random delay of the user $k$ 's signal reaching the receiver. The matched filter template signal of user $p(p=1,2, \ldots, K)$ in the matched filter of the receiver can be written 
as:

$$
m_{p}(t)=\sum_{j=0}^{N_{s}-1} c_{j}^{(p)} p\left(t-i T_{f}-j T_{c}-\tau_{p}\right)
$$

The output of the signal matching filter correlator of user $p$ can be expressed as:

$$
\begin{aligned}
y_{i}^{(p)} & =\int_{i T_{f}+\tau_{p}}^{(i+1) T_{f}+\tau_{p}} r(t) m_{p}(t) d t \\
& =\alpha_{p} \sqrt{P_{p}} E N_{s} d_{i}^{(p)}+\sum_{\substack{k=1 \\
k \neq p}}^{K} \alpha_{k} \sqrt{P_{k}} d_{i}^{(k)} \sum_{j=0}^{N_{s}-1} c_{j}^{(p)} c_{j}^{(k)} \int_{-\infty}^{\infty} p\left(t-j T_{c}-\tau_{p}\right) p\left(t-j T_{c}-\tau_{k}\right) d t+n_{p}
\end{aligned}
$$

where $\int_{i T_{f}+\tau_{p}}^{(i+1) T_{f}+\tau_{p}} r(t) m_{p}(t) d t$ denotes the matched filter output of the baseband information bits of user p. $\alpha_{p} \sqrt{P_{p}} E N_{s} d_{i}^{(p)}$ denotes the MAI to user $p$. Another item denotes the Gaussian white noise value after the matched filter.

\section{FPGA implementation of the GMM MUD algorithm}

The overview of the FPGA-based GMM MUD system block diagram is shown in Fig.3.

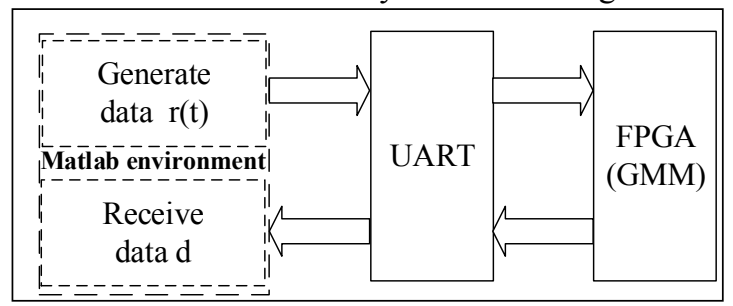

Fig. 3. Overview of the FPGA-based GMM MUD.

The FPGA hardware platform of multi-user detection algorithm based on Gaussian mixture model selects DE3 development board from Altera. The design chooses FPGA/Matlab joint debugging method based on serial interface. Using Matlab to generate signal $r(t)$ and receive data $d$. Hence, FPGA data transmission and reception are realized through RS232.

The FPGA block diagram for the GMM MUD algorithm as shown in Fig.4.

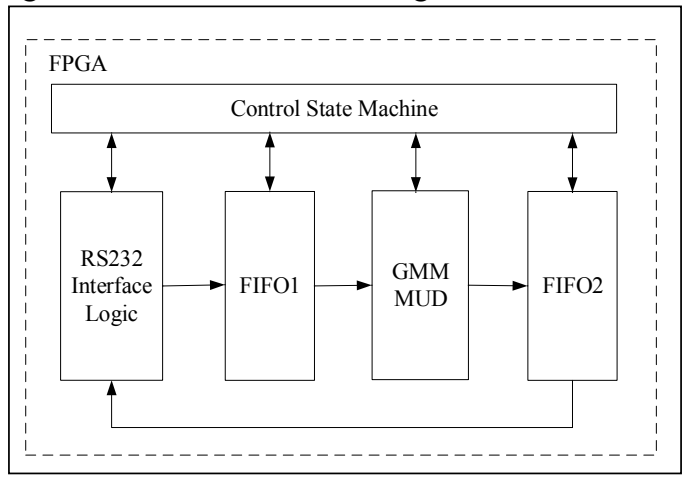

Fig. 4. FPGA block diagram for the GMM MUD algorithm. 


\subsection{GMM-based multi-user detector}

The GMM is a model formed by clustering several Gaussian probability density functions. GMM MUD structure block diagram is shown in Fig.5.

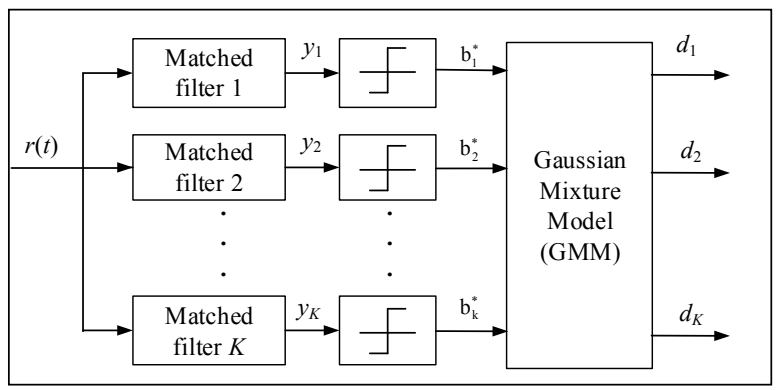

Fig. 5. GMM MUD structure block diagram.

The Gaussian mixture model can be defined as:

$$
p(\mathrm{x})=\sum_{i=1}^{K} w_{i} p\left(\mathrm{x} \mid \mu_{i}, \sigma_{i}^{2}\right)
$$

where $K$ denotes the number of models, $w_{i}$ denotes the weight of the $i$ th Gaussian model, and $\sum_{i=1}^{K} w_{i}=1 \cdot \mu_{i}$ denotes the mean of the $i$ th Gaussian model, and $\sigma_{i}^{2}$ denotes the variance of the $i$ th Gaussian model.

For a given number of models $K$, the GMM uses parameter estimation method to obtain the weight $w_{i}$, mean $\mu_{i}$, and variance $\sigma_{i}^{2}$ of each mixture component. The function can be written as:

$$
L=\prod_{j=i}^{m} \log \left(\sum_{i=1}^{K} w_{i} p\left(\mathrm{x} \mid \mu_{i}, \sigma_{i}^{2}\right)\right)
$$

However, it is generally used to solve by the expectation maximization (EM) algorithm. Solving process can be expressed as:

- According to the given $\mathrm{K}$ value, initialize the weight $w_{i}$, mean $\mu_{i}$ and variance $\sigma_{i}^{2}$.

- Expectation-step. Calculate the $i$ th sample $\mathrm{x}_{i}$, which is the posterior probability generated by the $k$ th model.

- Maximization-step. Update the mean and variance by the posterior probability obtained in E-step.

- Repeat E-step and M-step until the likelihood function increment is less than the convergence threshold.

\subsection{Control state machine}

The key task of Control State Machine is drive the other sub-modules, in this way to control the system work orderly. The state-transition diagram is shown in Fig.6. 


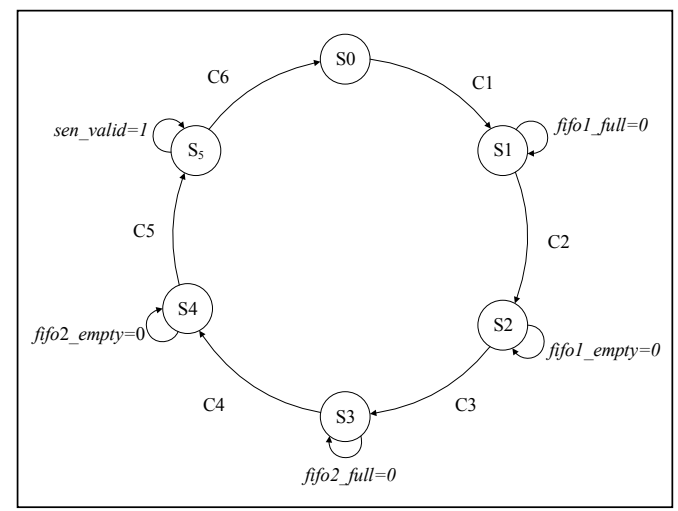

Fig. 6. State-transition diagram.

- SO: System initial state.

- C1: Received data valid.

- S1: FIFO1 receive data .

- C2: FIFO1 is full.

- $S 2$ : Read and process data from FIFO1.

- C3: FIFO1 is empty.

- S3: Write the processed data to FIFO2.

- $C 4$ : FIFO2 is full.

- S4: Send the data in FIFO2 .

- C5: FIFO2 is empty.

- S5: Clear all.

- C6: Receive data valid signal enable.

\section{Implementation results and analysis}

In this section, we set the number of users in the communication system to 10 , the SNR ranging from $0 \mathrm{~dB}$ to $10 \mathrm{~dB}$ spaced $1 \mathrm{~dB}$, the serial port baud rate is 230400 , and send 500000 modulation code elements. The BER performance of the GMM-based multi-user detector is shown in Fig. 7.

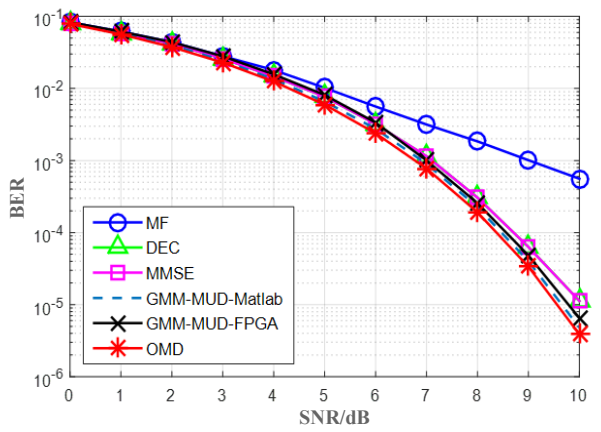

Fig.7. The result of BER performance. 
We present result to show the detection performance of the GMM-based multi-user detector. In Fig.7, it can be seen that the performance of FPGA measured bit error rate based on Gaussian Mixture Model multi-user detection is lower than the theoretical bit error rate. When the SNR is low, the BER performance of the GMM-based multi-user detector is close to DEC multi-user detection and MMSE multi-user detection. When the SNR is high, the BER performance of the GMM-based multi-user detector is better than DEC multi-user detection and MMSE multi-user detection.

\section{Summary}

The FPGA implementation of GMM MUD algorithm have been proposed in this paper. The processing speed of the design is faster than the theoretical algorithm. While ensuring the capability, the algorithm has lower complexity, faster speed and real-time performance. Furthermore, the system is more implementable.

The research in this article was supported by the National Natural Science Foundation of China (grant numbers 61871157, 61471142, and 61102084).

\section{References}

1. Sidibeh K., Vladimirova T, IEEE 802.11 Optimization Techniques for Inter-Satellite Links in LEO Networks, The 8th International Conference on Advanced Communication Technology, 2006, pp.1177-1182.

2. Oleski P. J., Patton R. W., Bharj S.,Thaduri M.,Transmission Receive Model for Space Ground Link Subsystem (SGLS) and Unified S-band (USB) satellite telemetry, tracking and commanding (TT\&C) and communications, IEEE Military Communications Conference, 2004, pp. 880-885.

3. Xiaoya Zuo, Ding Wang, Interference cancelation for non-CP DS-UWB system with MRC frequency domain equalization, Proceedings of the 2015 Third International Conference on Computer, Communication, Control and Information Technology (C3IT),2015, pp.1-4.

4. Zhoujin Sun, Qiuming Zhang, Yanjun Majun, Multiple Access DS-UWB Using Linear Chirp Encoding Waveforms, 2016 3rd International Conference on Information Science and Control Engineering (ICISCE), 2016, pp.1366-1369.

5. Nguyen Thi Huyen, Pham Thanh Hiep, Proposing adaptive PN sequence length scheme for testing non-destructive structure using DS-UWB, 2019 3rd International Conference on Recent Advances in Signal Processing,Telecommunications \& Computing (SigTelCom), 2019, pp.10-14.

6. Taoufiq Ait Benrami, Younes Jabrane, Jilali Antari, Radouane Iqdour, Wavelet Networks for MAI cancellation in MC-DS/CDMA, 2016 5th International Conference on Multimedia Computing and Systems (ICMCS),2016, pp.519 - 522.

7. Mangala R. Gajbhiye, Jitendriya Kumar Satapathy, Performance comparison of adaptive MMSE-MRC MUD for DS-CDMA system in different multipath channels, Proceedings of the 2011 International Conference on Communication, Computing \& Security(ICCCS), 2011, pp.56-61.

8. Kenneth Schneider, Optimum detection of code division multiplexed signals, IEEE Transactions on Aerospace and Electronic Systems, 1979,15(1):181-185. 
9. Bo Li, Qingzhi Liu, Zhendong Yin, Zhilu Wu, A novel SNR estimator for DS-UWB wireless sensor network, 2017 2nd International Conference on Computer, Mechatronics and Electronic Engineering (CMEE),2017, pp.87-93.

10. Xiaofei Li, Di He, HSBCS framework based performance detection for DS-UWB Systems, The Journal of Networks, Software Tools and Applications, 2019,22(2):26552665.

11. C. T. Chiang, A. C. Chang, DOA estimation in the asynchronous DS-CDMA system, IEEE Transactions on Antennas and Propagation, 2003,51(1):40 - 47. 